\title{
Target-selective homologous recombination cloning for high-throughput generation of monoclonal antibodies from single plasma cells
}

\author{
Nobuyuki Kurosawa', Megumi Yoshioka ${ }^{2}$ and Masaharu Isobe ${ }^{3 *}$
}

\begin{abstract}
Background: Molecular cloning of functional immunoglobulin genes from single plasma cells is one of the most promising technologies for the rapid development of monoclonal antibody drugs. However, the proper insertion of PCR-amplified immunoglobulin genes into expression vectors remains an obstacle to the high-throughput production of recombinant monoclonal antibodies.

Results: We developed a single-step cloning method, target-selective homologous recombination (TS-HR), in which PCR-amplified immunoglobulin variable genes were selectively inserted into vectors, even in the presence of nonspecifically amplified DNA. TS-HR utilizes Red/ET-mediated homologous recombination with a target-selective vector (TS-vector) with unique homology arms on its termini. Using TS-HR, immunoglobulin variable genes were cloned directly into expression vectors by co-transforming unpurified PCR products and the TS-vector into E. coli. Furthermore, the high cloning specificity of TS-HR allowed plasmids to be extracted from pools of transformed bacteria without screening single colonies for correct clones. We present a one-week protocol for the production of recombinant mouse monoclonal antibodies from large numbers of single plasma cells.

Conclusion: The time requirements and limitations of traditional cloning procedures for the production of recombinant immunoglobulins have been significantly reduced with the development of the TS-HR cloning technique.
\end{abstract}

\section{Background}

Molecular cloning of immunoglobulin variable $(\mathrm{V})$ genes from single isolated plasma cells is a powerful tool for the unbiased generation of recombinant monoclonal antibodies [1-3]. Fluorescence-activated single-cell sorting followed by a single-cell reverse-transcription polymerase chain reaction (RT-PCR) has been shown to enable the high-throughput production of $\mathrm{V}$ gene DNA fragments [4-6]. However, the proper insertion of the $\mathrm{V}$ gene DNA fragments into expression vectors remains an obstacle to the high-throughput production of recombinant monoclonal antibodies.

The most commonly employed cloning method for introducing $\mathrm{V}$ gene DNA fragments into vectors is

\footnotetext{
* Correspondence: isobe@eng.u-toyama.ac.jp

${ }^{3}$ Laboratory of Molecular and Cellular Biology, Faculty of Science and Engineering, Graduate School, University of Toyama, 3190 Gofuku, Toyamashi, Toyama, 930-8555, Japan

Full list of author information is available at the end of the article
}

ligation-dependent cloning $[1,3,5,6]$. However, ligationdependent cloning is often hampered by the requirement for multiple rounds of enzyme treatments and purification of both the inserts and vectors. Furthermore, the limited number of appropriate restriction enzyme sites in the insert and vector DNA limits flexibility in constructing recombinant molecules. Recently, site-specific recombination-based cloning has emerged as a ligation-independent cloning method [7-9]. However, this technology introduces extra codons into the gene's primary sequence at the site of recombination, which may interfere with the folding and stability of the resulting protein. In contrast, homologous recombination technologies enable the seamless insertion of any DNA fragment at any desired position [10]. Recently, In-Fusion homologous recombination, which can join a DNA fragment and a linear vector with 15 bases of homology at their ends, was used in the production of recombinant monoclonal antibodies [11]. Although the

\section{C) Biomed Central}


In-Fusion technology offers several advantages as a high-throughput procedure, the amplified $\mathrm{V}$ gene DNA fragments must be purified to remove salts, primers and nonspecifically amplified DNA before the reaction. Subsequent steps are also necessary to screen single colonies for a correct clone, which can result in additional labor and increased cost. Thus, a method for the highthroughput cloning of PCR-amplified V gene DNA fragments into vectors that bypasses these tedious preliminary steps is needed.

We have developed a method, termed target-selective homologous recombination (TS-HR), in which PCRamplified $\mathrm{V}$ gene DNA fragments can be selectively cloned into vectors, even in the presence of nonspecifically amplified PCR products. This system, together with the additional methods described herein, circumvents the problems associated with the amplification and cloning of $\mathrm{V}$ gene DNA fragments and provides a system for the high-throughput production of recombinant mouse monoclonal antibodies from large numbers of single plasma cells within a one-week time span (Figure 1).

\section{Results and Discussion}

Low cloning specificity in conventional, Red/ET-mediated homologous recombination

Red/ET-mediated recombination is a powerful homologous recombination system based on the function of either the Red operon of lambda phage or RecE/RecT from Rac phage [12-15]. To evaluate the cloning specificity of conventional Red/ET-mediated homologous recombination, we conducted a pilot experiment using a linear nonselective vector (NS-vector) and an artificially amplified $\mathrm{V}$ gene and mock DNA. The V gene was composed of an upper primer-derived sequence (P1), a poly$\mathrm{dG} / \mathrm{dC}$ sequence (T1), a human $\mathrm{VH}$ sequence, part of the constant gene sequence (T2) and a lower primerderived sequence (P2). The mock DNA was composed of the P1 sequence, part of the GFP sequence and the P2 sequence. The linear NS-vector contained homology arms at its ends (VP1 and VP2) that were homologous to the P1 and P2 sequences, respectively (Figure 2A, left). When the $\mathrm{V}$ gene and the mock DNA were mixed in a 1:1 molar ratio and introduced into competent cells with the NS-vector, each DNA fragment was inserted into the NS-vector with the same probability (Figure 2B, upper left). Transformation of the bacteria with the $\mathrm{V}$ gene and the mock DNA fragment in a 1:4 molar ratio resulted in the insertion of the $\mathrm{V}$ gene in $25 \%$ of the clones (10 out of 40 clones) (Figure 2B, lower left). Because the conventional homologous recombination reaction was mediated through the primer-derived sequences, the DNA fragments containing the primer sequences at their ends were non-selectively inserted into the NS vector.

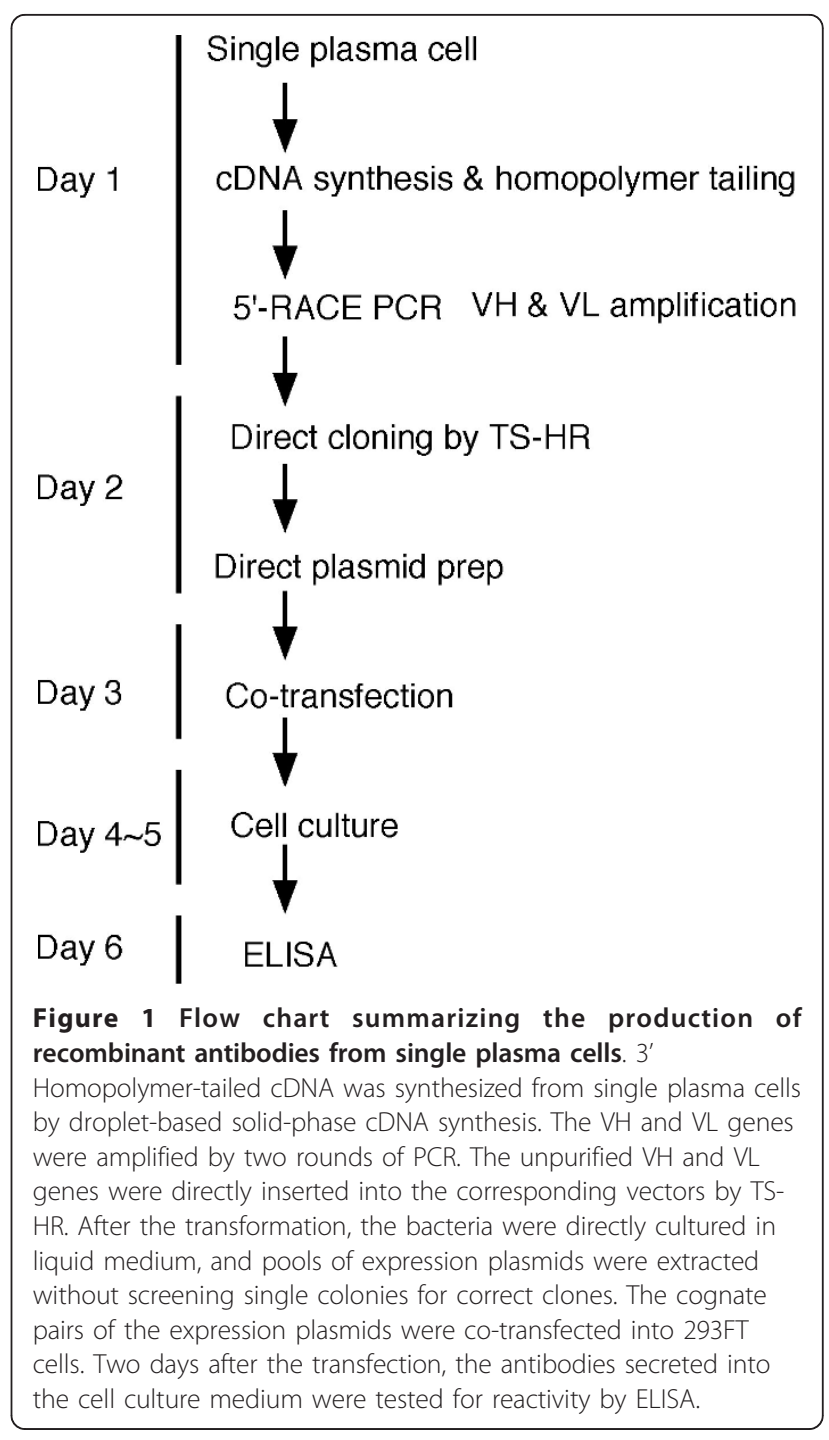

\section{High cloning specificity of TS-HR}

To selectively clone the $\mathrm{V}$ gene, even in the presence of nonspecific amplified DNA, we constructed a new vector: the target-selective vector (TS-vector). The TS-vector has homology arms (VP1-VT1 and VP2-VT2) on its ends. The VP1 (VP2) sequence shares homology with the P1 (P2) sequence, which is a primer-derived sequence. VT1 (VT2) shares homology with T1 (T2), which is a part of the $\mathrm{V}$ gene-specific sequence internal to P1 (P2) (Figure 2A, right). When the V gene and the mock DNA were mixed in a 1:1 molar ratio and introduced into competent cells with the linear TS-vector, $92.5 \%$ of the analyzed colonies contained the $\mathrm{V}$ gene (Figure 2B, upper right). Transformation of the bacteria with the $\mathrm{V}$ gene and the mock DNA in a 1:4 molar ratio resulted in the insertion of the $\mathrm{V}$ gene in $72.5 \%$ of the analyzed colonies (29 out of 40 clones) (Figure 2B, lower right). This result confirms the high cloning 


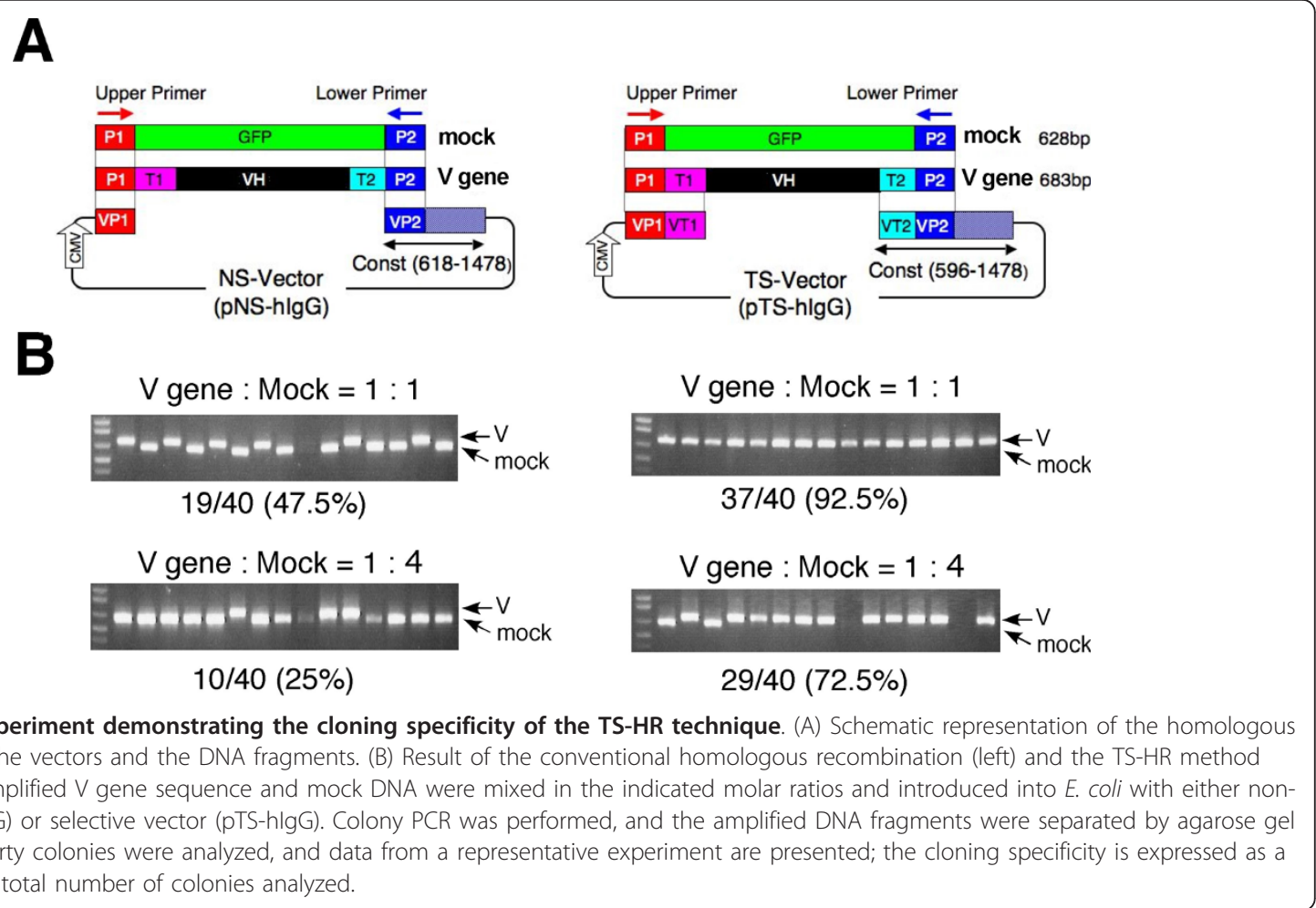

specificity of the TS-HR method, even in the presence of an excess amount of nonspecific amplified DNA.

The mechanism for selective cloning in the TS-vector is illustrated in Figure 3. The 5' ends of the linear DNA are removed by the Red $\alpha$ 5' $\rightarrow 3$ ' exonuclease in E. coli, resulting in the generation of 3 ' single-stranded tails from the TS-vector, target and non-target DNA. When the single-stranded tails of the target DNA anneal to the complementary single-stranded tails of the TS-vector through the long homology overlaps (P1-T1 to VP1-VT1 and P2$\mathrm{T} 2$ to VP2-VT2), the recombination reaction proceeds via route $\mathrm{I}$. When the single-stranded tails of the mock DNA anneal to the complementary single-stranded tails of the TS-vector through the short homology overlaps (P1 to VP1 and P2 to VP2), the recombination reaction proceeds via route III. However, the route I reaction dominates over the route III reaction because the homologous recombination reaction depends on the length of the homology overlap. The homologous recombination reaction also proceeds when the single-stranded tails of the TS-vector anneal to the complementary singlestranded tails of the target DNA (Figure 3, route II). However, the homologous recombination reaction does not proceed when the single-stranded tails of the TS-vector anneal to the single-stranded tails of the mock DNA. The 3' ends of the single-stranded tails of the TS vector (VT1 and VT2) fail to serve as primers for strand extension due to the 3' end mismatches (Figure 3, route IV).

\section{Application of TS-HR for high-throughput production of} recombinant mouse antibodies

To demonstrate the usefulness of TS-HR, we amplified the variable region of the immunoglobulin heavy chain $(\mathrm{VH})$ and the variable region of the immunoglobulin light chain $(\mathrm{VL})$ genes from single mouse plasma cells by 5 ' RACE PCR and attempted to insert the fragments into the expression vectors. The pairs of $\mathrm{VH}$ and $\mathrm{VL}$ genes were successfully amplified from single plasma cells (Figure 4A). When conventional Red/ET-mediated homologous recombination was conducted with a nonselective vector (pNS-mIgG) and unpurified PCR products from lane 9 or 13, only $50 \%$ and 30\% of the colonies contained the $\mathrm{VH}$ gene were obtained, respectively (Figure 4B, upper). These results clearly indicate that purification of the $\mathrm{V}$ gene is required prior to the conventional homologous recombination reaction. However, when TS-HR was conducted with a V gene-selective vector (pTS-mIgG) and unpurified PCR products from lane 9 or $13,80 \%$ and $90 \%$ of the colonies contained the $\mathrm{VH}$ gene were obtained, respectively (Figure 4B, lower). Theses results were in good agreement with the pilot experiment in Figure 2.

In light of the high cloning specificity of the TS-HR technique, we prepared the immunoglobulin expression plasmids without screening single colonies for a correct clone. When the pool of plasmids was extracted from the bacteria directly cultured in liquid medium after 


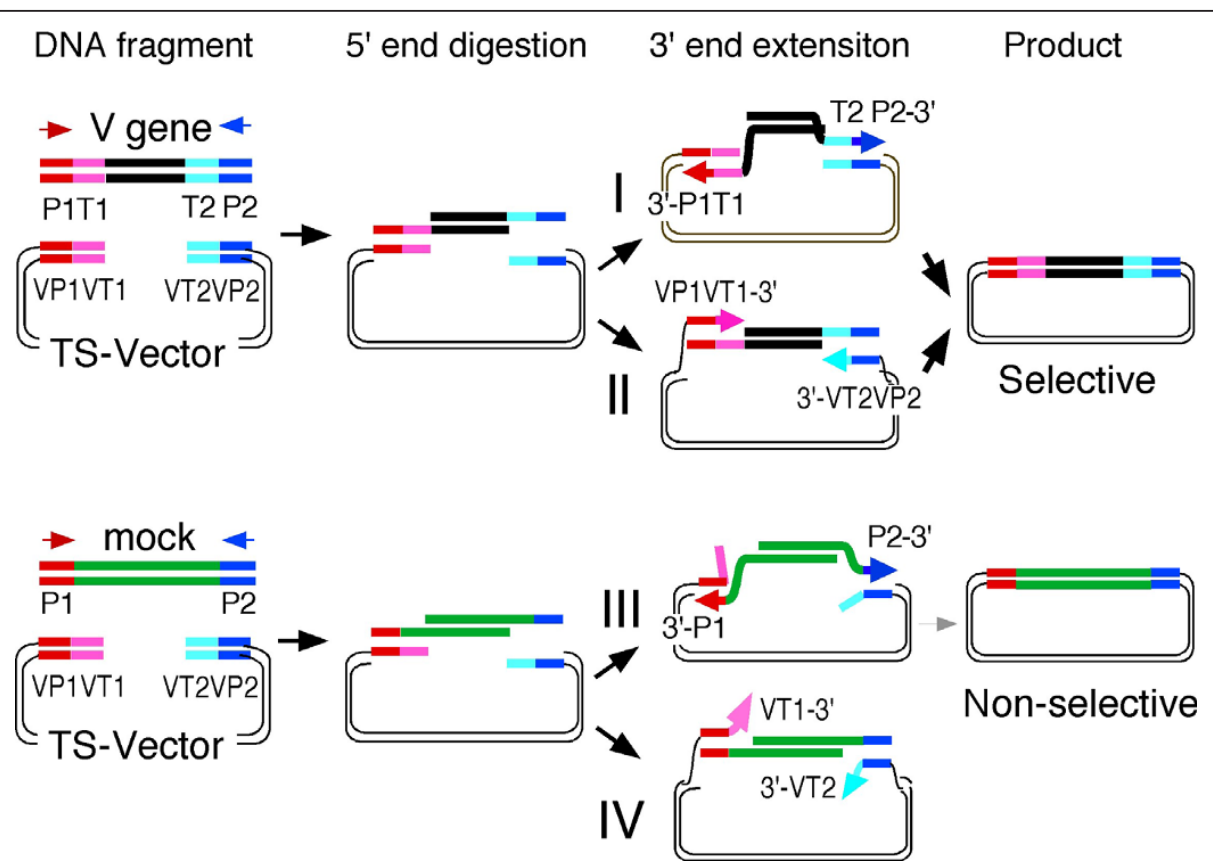

Figure 3 Schematic illustration of TS-HR. In E. coli, linear DNA was digested by the Reda $5^{\prime} \rightarrow 3^{\prime}$ exonuclease, resulting in the generation of $3^{\prime}$ single-stranded tails. Homologous recombination of the $V$ gene strands and the TS-vector, which is mediated by the long overlapping sequences ( $50 \mathrm{bp}$ ), proceeds by $3^{\prime}$ end extension from P1 and P2 (route I). However, homologous recombination of the mock DNA strands and the TS-vector is inefficient because of their short overlapping sequences (route III). Homologous recombination of the TS-vector and the V gene, which is mediated through the long overlapping sequences ( 50 bp), proceeds by $3^{\prime}$ end extension from VT1 and VT2 (route II). However, homologous recombination of the TS vector and the mock DNA does not occur because strand extension from VT1 and VT2 is blocked by $3^{\prime}$ end mismatches (route IV).

transformation, all 40 examined plasmids contained an insert of the expected size (Figure 4C). Furthermore, the $\mathrm{V}$ gene DNA sequence was present in all of the plasmids examined, and the cloning reaction proceeded as expected, with no insertions or deletions at the cloning junctions (Figure 4D and Table 1). We also observed the successful in-frame insertion of $\mathrm{VH}$ genes belonging to G1, G2a and G2b, indicating the unbiased insertion of the IgG subclass (Table 1).

Co-transfection of the cognate pair of heavy chain and light chain expression plasmids into 293FT cells resulted in the secretion of recombinant antibodies (19 out of $20)$; seven clones specifically reacted with the antigen (Figure 5).

The major advantages of the TS-HR system include its specificity, rapidity and robustness. By using the TS-HR technique, the time-consuming processes inherent in current cloning methods, such as the purification of the $\mathrm{V}$ gene DNA fragment and the plating of transformed bacteria to screen single colonies for correct clones, are dramatically reduced. Due to the simplicity of our system, dozens of expression plasmids can be produced simultaneously, enabling us to produce recombinant antibodies from large numbers of single plasma cells. In addition, unlike other cloning systems, Red/ET-mediated recombination can be performed inexpensively in large quantities, saving investigators significant time and expense. The TS-HR system can be applied to the selective cloning of DNA fragments in which primer regions and their internal gene-specific sequences are constant but the intervening regions vary; $\mathrm{T}$-cell receptor genes are an example of such a target sequence.

\section{Conclusion}

The method described here overcomes several major obstacles to the cloning of PCR-amplified V gene DNA fragments into expression vectors. First, unlike currently employed cloning techniques, the TS-HR method does not require purification of the V gene. Second, TS-HR does not require the screening of transformed bacterial colonies for correct clones. These features suggest that TS-HR is directly applicable to high-throughput applications.

\section{Methods}

\section{Materials}

The animal experiments in this study were approved by the Committee on Animal Experimentation at the University of Toyama. Seven-week-old female ICR mice were immunized twice (at intervals of one month) 
A
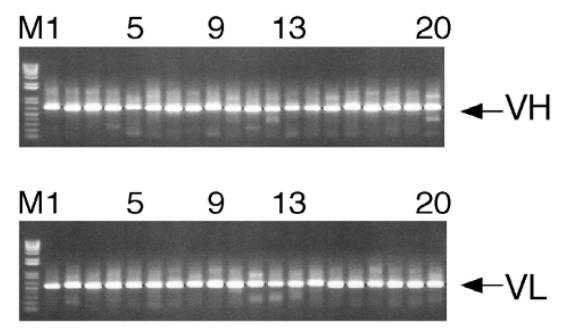

C

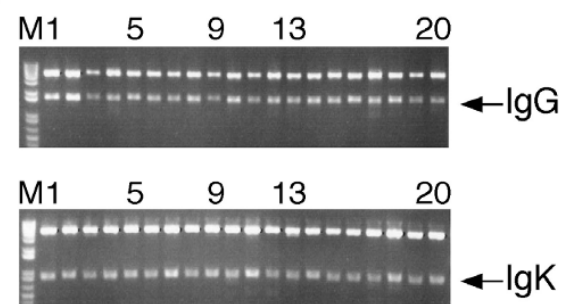

B

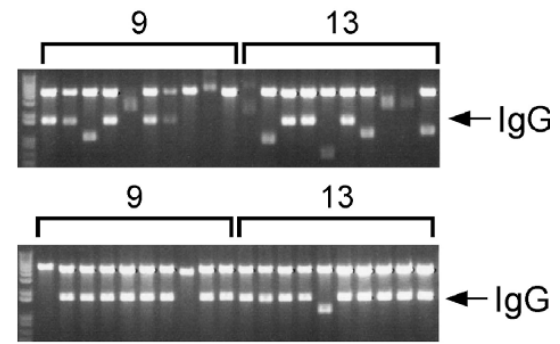

D

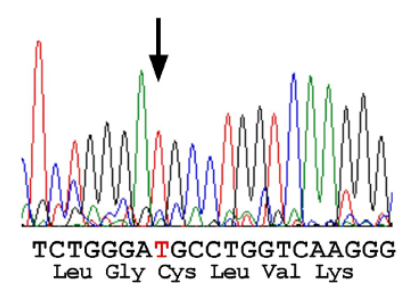

Figure 4 High-throughput production of recombinant mouse monoclonal antibodies by the TS-HR technique. (A) Amplification of cognate pairs of $\mathrm{VH}$ and $\mathrm{VL}$ genes from single plasma cells by 5' RACE PCR. (B) The PCR product obtained (A, upper, lane 9 and 13) was directly inserted into pNS-mlgG by conventional homologous recombination (upper) or into pTS-mlgG by TS-HR (lower). After the transformation, bacteria were plated on agar plates containing $1 \%$ sucrose and kanamycin. Ten colonies were cultured, and plasmids were extracted from the overnight culture. Plasmids digested with Sall/Notl were electrophoresed in a 1\% agarose gel. (C) The amplified VH and VL genes obtained in (A) were directly cloned into linear pTS-mlgG and pTS-mlgK, respectively, by TS-HR. After the transformation, bacteria were directly inoculated into LB medium containing $1 \%$ sucrose and $50 \mu \mathrm{g} / \mathrm{ml}$ of kanamycin, and pools of plasmids were prepared from the overnight culture. Plasmids digested with Sall/Notl were electrophoresed in a 1\% agarose gel. (D) DNA sequencing of the plasmid obtained in (C) confirmed the in-frame insertion of the $V$ gene into the plasmid. A representative sequence of data from the plasmid originating from $(C$, upper) lane 9 is shown; the arrow indicates the DNA fragment-vector junction of the Ig constant gene.

Table 1 V-(D)-J repertoire of cloned $\mathrm{V}$ genes.

\begin{tabular}{|c|c|c|c|c|c|c|}
\hline & \multicolumn{4}{|c|}{ Gamma Chain } & \multicolumn{2}{|c|}{ Kappa Chain } \\
\hline & V & $\mathrm{D}$ & $J$ & isotype & v & $J$ \\
\hline 1 & IGHV10-1*01 & IGHD2-4*01 & IGHJ3*01 & $\mathrm{G} 2 \mathrm{~b}$ & IGKV6-32*01 & IGKJ5*01 \\
\hline 2 & IGHV1-5*01 & IGHD2-4*01 & $\mid \mathrm{GHJ} 1 * 03$ & G2a & IGKV1-117*01 & $\mid G K J 4^{*} 02$ \\
\hline 3 & IGHV1-4*01 & IGHD1-1*02 & IGHJ1*03 & G1 & IGKV6-15*01 & $|G K| 5^{*} 01$ \\
\hline 4 & IGHV1-26*01 & IGHD4-1*02 & IGHJ2*01 & $\mathrm{G} 2 \mathrm{~b}$ & IGKV6-17*01 & $\mid G K J 1 * 01$ \\
\hline 5 & IGHV5-17*01 & IGHD2-3*01 & $\mid \mathrm{GHJ} 3 * 01$ & $\mathrm{G} 2 \mathrm{~b}$ & IGKV5-43*01 & $\mid G K J 5 * 01$ \\
\hline 6 & IGHV4-1*01 & IGHD3-2*01 & $\mid G H J 2^{*} 01$ & G1 & IGKV6-23*01 & $\mid G K J 4^{*} 01$ \\
\hline 7 & IGHV1-22*01 & IGHD2-12*01 & IGHJ3*01 & G1 & IGKV14-111*01 & $\mid G K J 4^{*} 01$ \\
\hline 8 & IGHV1-26*01 & IGHD2-2*01 & IGHJ3*01 & G1 & IGKV4-78*01 & $\mid G K J 1 * 01$ \\
\hline 9 & IGHV5-17*01 & IGHD2-3*01 & IGHJ3*01 & $\mathrm{G} 2 \mathrm{~b}$ & IGKV5-43*01 & $\mid G K J 5 * 01$ \\
\hline 10 & IGHV5-16*01 & IGHD3-2*01 & $\mid G H J 2^{*} 01$ & G1 & IGKV6-20*01 & $\mid G K J 4^{*} 02$ \\
\hline 11 & IGHV1-19*01 & IGHD4-1*01 & $\mid \mathrm{GHJ} 2^{*} 01$ (a) & G1 & IGKV1-135*01 & IGKJ2*03 \\
\hline 12 & IGHV1-9*01 & Not identified & $\mid G H J 4^{*} 01$ & $\mathrm{G} 2 \mathrm{~b}$ & IGKV1-99*01 & $\mid G K J 1 * 01$ \\
\hline 13 & IGHV1-62-2*01 & IGHD1-1*01 & $\mid G H J 2^{*} 01$ (a) & G2a & IGKV12-46*01 & $|G K| 1 * 01$ \\
\hline 14 & IGHV1-70*01(p) & IGHD3-1*01 & $\mid G H J 4^{*} 01$ & G1 & IGKV1-117*01 & $\mid G K J 2^{*} 01$ \\
\hline 15 & IGHV8-12*01 & IGHD1-1*01 & $\mid G H J 1 * 01$ & G1 & IGKV10-96*01 & $|G K| 1 * 01$ \\
\hline 16 & IGHV5-4*01 & IGHD4-1*01 & IGHJ1*03 & G1 & IGKV10-94*03/08 & $\mid G K J 1 * 01$ \\
\hline 17 & IGHV1-53*01 & IGHD1-1*01 & $\mid G H J 2 * 01$ & $\mathrm{G} 2 \mathrm{~b}$ & IGKV12-41*01 & $\mid G K J 1 * 01$ \\
\hline 18 & IGHV1-22*01 & IGHD2-12*01 & IGHJ3*01 & G1 & IGKV14-111*01 & IGKJ4*01 or 02 \\
\hline 19 & IGHV1-66*01 & |GHD1-1*01 & $\mid \mathrm{GHJ} 2^{*} 01$ (a) & G1 & IGKV5-45*01 & IGKJ2*03 \\
\hline 20 & IGHV5-16*01 & IGHD3-3*01 & $\mid G H J 1 * 03$ & $\mathrm{G} 2 \mathrm{~b}$ & IGKV1-115*01(p) & $\mid G K J 5^{*} 01$ \\
\hline
\end{tabular}

Pools of plasmids originating as described in Figure $4 \mathrm{C}$ were sequenced, and their $\mathrm{V}-(\mathrm{D})-\mathrm{J}-(\mathrm{H})$ repertoires were determined. 

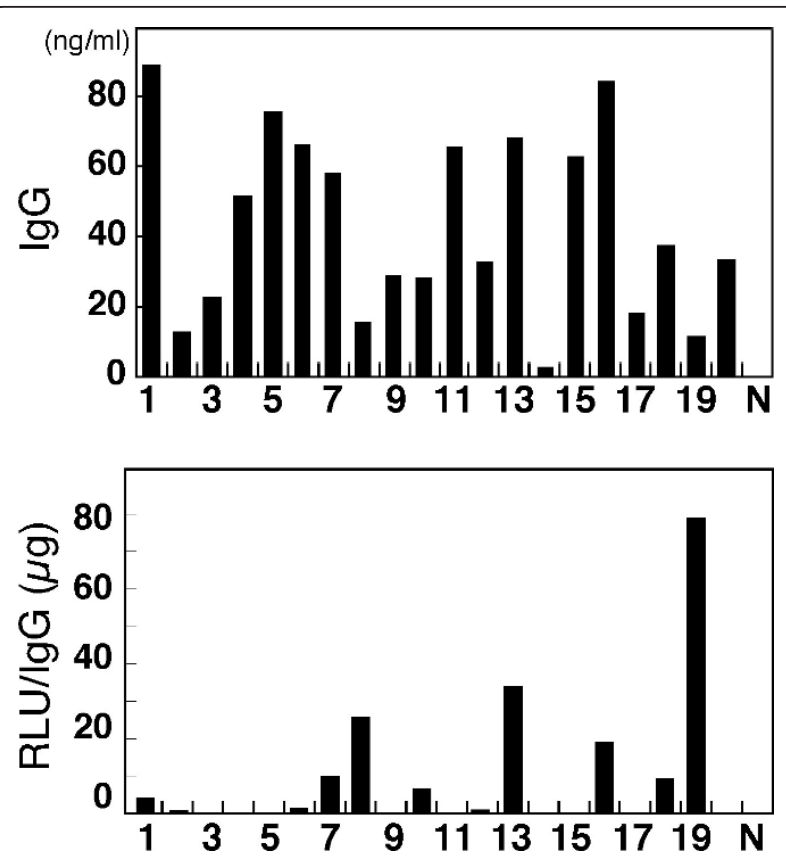

Figure 5 Expression of recombinant mouse antibodies. (A) The concentration of the recombinant antibodies in the supernatant of cultured 293FT cells was determined by sandwich ELISA. N indicates a negative control; all of the incubation steps were identical, except that non-transfected cell culture medium was used. (B) The specific activity of the recombinant antibodies was expressed as RLU/s/lgG ( $\mu \mathrm{g})$.

intradermally in the footpad with $50 \mu \mathrm{l}$ of a 50:50 waterin-oil emulsion containing $25 \mu \mathrm{g}$ of egg albumin (Nakarai Tesque). Two to four weeks after the second immunization, popliteal lymph nodes were surgically removed, and plasma cells were isolated using the CD138+ plasma cell isolation kit according to the manufacturer's instructions (Miltenyi Biotec). The isolated plasma cells were microscopically identified after staining with an anti-CD138 antibody. Single cells were captured with a standard micromanipulator mounted on an inverted microscope and suspended in $3 \mu \mathrm{l}$ of a lysis/ binding solution (100 mM Tris $\mathrm{HCl} \mathrm{pH} \mathrm{7.5,} 500 \mathrm{mM}$ $\mathrm{LiCl}, 1 \%$ lithium dodecyl sulfate and $5 \mathrm{mM}$ dithiothreitol) containing $3 \mu \mathrm{g}$ of oligo-dT magnetic beads.

\section{Synthesis of 3' homopolymer-tailed cDNA from single cells}

3' Homopolymer-tailed cDNA from single plasma cells was prepared by a droplet-based solid-phase cDNA synthesis termed Magnetic-beads Reaction through Arrayed Hanging Drops (MAGrahd), which is described in more detail below. Briefly, $3 \mu \mathrm{l}$ each of single-cell lysate containing oligo-dT magnetic beads, washing solution 1 (10 mM Tris $\mathrm{HCl}$ pH 7.5, $150 \mathrm{mM} \mathrm{LiCl,} \mathrm{0.1 \%}$ lithium dodecyl sulfate and $1 \mathrm{mM}$ EDTA), washing solution 2 (75 mM KCl, $3 \mathrm{mM} \mathrm{MgCl}_{2}, 0.1 \%$ Triton X-100, 5
mM DTT and 2 units of RNase inhibitor), reverse transcription solution (washing solution 2 containing 10 units of SuperScript III reverse transcriptase), washing solution 3 (50 $\mathrm{mM}$ potassium phosphate $\mathrm{pH} 7.0,0.1 \%$ Triton $\mathrm{X}-100$ and $4 \mathrm{mM} \mathrm{MgCl}_{2}$ ), terminal transferase solution (washing solution 3 containing 10 units of terminal deoxynucleotidyl transferase), washing solution 4 (TE buffer containing $0.1 \%$ Triton X-100) and PCR solution (1x ExTaq buffer containing $0.1 \mathrm{mM}$ dNTPs and $0.1 \%$ Triton $\mathrm{X}-100$ ) were deposited on the surface of a hydrophobic film to form a droplet.

The mRNA immobilized on the magnetic beads was extracted from the cell lysis droplet with an externally applied magnetic force and sequentially transferred into droplets of washing solution 1, washing solution 2 and the reverse transcription solution. After 15 minutes of reverse transcription at $37^{\circ} \mathrm{C}$, the cDNA immobilized on the magnetic beads was sequentially transferred into a droplet of washing solution 3 and a droplet of terminal transferase solution for 3' poly-dG tailing. After the terminal transferase reaction had proceeded for $15 \mathrm{~min}$ utes at $37^{\circ} \mathrm{C}$, the homopolymer-tailed cDNA immobilized on the magnetic beads was sequentially transferred into droplets of washing solution 4 and PCR solution.

\section{Amplification of $\mathrm{VH}$ and $\mathrm{VL}$ genes from single plasma cells}

DNA fragments of the $\mathrm{VH}$ and $\mathrm{VL}$ chain genes from single mouse plasma cells were amplified by the rapid amplification of the 5' cDNA ends by polymerase chain reaction (5' RACE-PCR) [16]. Briefly, the first round of PCR was performed with a forward primer (AP3dC-S) specific for the homopolymer-tail and reverse primers specific for the respective immunoglobulins' gamma and kappa constant regions (mIgG1st-AS and mIgK1st-AS). The second round of PCR was performed with a forward primer (MCSAP3-S) that annealed to the first PCR forward primer and with the respective nested reverse primer specific for the IgG (mIgG2nd-AS) or IgK (mIgK2nd-AS) constant regions. The PCR primers used for the amplification of the mouse $\mathrm{VH}$ and VL genes are detailed in Table 2. PCR was performed using the ExTaq DNA polymerase in a BIO-RAD MyCycler (35 cycles of reaction at $94^{\circ} \mathrm{C}$ for 30 seconds, $68^{\circ} \mathrm{C}$ for 40 seconds and a final extension at $72^{\circ} \mathrm{C}$ for 3 minutes).

\section{Plasmid construction}

A human IgG constant region was amplified by RT-PCR with human B cell cDNA as a template. An 882-bp DNA fragment encoding the human IgG constant gene (596-1478 of the nucleotide accession number AK301389) was amplified by PCR with the primer 596/ 617-S (5'- GCCCGGGATCCGATATCACGTGGAACTCAGGCGCCCTGACC-3', restriction enzyme sequences 
Table 2 Primers used for amplifying the mouse VH and VL genes.

\begin{tabular}{lll}
\hline Use & Primer & Sequence \\
\hline 1st PCR upper & AP3dC-S & CGGTACCGCGGGCCCGGGATCCCCCCCCCCCCCDN \\
\hline 2nd PCR upper & MCSAP3-S & CTTCGAATTCTGCAGTCGACGGTACCGCGGGCCCGGGA \\
\hline $\operatorname{lgG}$ 1st PCR lower & mlgG1st-AS & ACCYTGCATTGAACTCCTTGCC \\
\hline $\operatorname{lgG}$ 2nd PCR lower & mlgG2nd-AS & CTGGACAGGGATCCAGAGTTCCA \\
\hline IgK 1st PCR lower & mlgK1st-AS & ACTGCCATCAATCTTCCACTTGACA \\
\hline IgK 2nd PCR lower & mlgK2nd-AS & ACTGAGGCACCTCCAGATGTAACT \\
\hline
\end{tabular}

underlined) and 1478-AS (5'-GAGTCGCGGCCGCCGT CGCACTCATTTACCCGGAG-3'). The amplified gene was digested with BamHI and NotI and subcloned into the BamHI/NotI sites of the pDsRed2-N1 vector (Clontech) to construct phIgG-C. The $s a c B$ gene was amplified from the pDNR1 vector (Clontech) by PCR with the primers 5'-CCCGATATCGATCCGACGTCCACATATACC-3' and 5'-CACGTGATATCGGCATTTTCT TTTGC-3', digested with EcoRV and inserted into the EcoRV site of phIgG-C to construct pIn-hIgG. The $\mathrm{dC13/dG13} \mathrm{linker} \mathrm{DNA} \mathrm{was} \mathrm{generated} \mathrm{by} \mathrm{annealing} \mathrm{the}$ oligonucleotides 5'-GATCCCCCCCCCCCCCGATATC3' and 5'-GATCGATATCGGGGGGGGGGGG -3' and inserting the fragment into the BamHI site of phIgG-C to produce the V gene-selective vector pTS-hIgG. A non-selective vector, pNS-hIgG, was amplified from pTS-hIgG by PCR with the primers 618/642-S (5'CAGCGGCGTGCACACCTTCCCGGCT-3') and AP3 MCS-AS (5'-TCCCGGGCCCGCGGTACCGTCGACTG CAGAATTCGAAG-3').

The mock DNA fragment and the V gene DNA fragment used for the pilot experiments were amplified by PCR using plasmids as templates; the primers used were an upper primer, MCSAP3-S and a lower primer, 618/ 642-AS (5'- AGCCGGGAAGGTGTGCACGCCGCTG -3 '). The mock fragment ( $628 \mathrm{bp}$ ) consisted of the upper primer-derived sequence (P1 region), part of the GFP gene sequence (non-target DNA) and the lower primer-derived sequence (P2 region). The target DNA fragment (683 bp) consisted of the upper primer-derived sequence ( $\mathrm{P} 1$ region), the poly-dC/dG sequence ( $\mathrm{T} 1$ region), the human immunoglobulin $\mathrm{VH}$ region, part of the human immunoglobulin gamma heavy chain constant region that included nucleotides 596-617 (T2 region) and the lower primer-derived sequence (P2 region). The amplified DNA fragments were purified by S-400 spin column. A schematic illustration of the DNA fragment and the vectors is shown in Figure 2A.

A DNA fragment encoding $901 \mathrm{bp}$ of the mouse IgG constant region (559-1460 of nucleotide accession number AB097849) was amplified with the primers 5'GATATCACGTGTGCCTGGTCAAGGGCTATTTCC CTGAG-3' (restriction site underlined) and 5'-CT CCGCGGCCGCTGGGATCATTTACCAGGAGAGT-3'.
A DNA fragment encoding 309 bp of the mouse IgK constant region (441-750 of nucleotide accession number AF466770) was amplified with the primers 5'-GATATCACGTGCTGTATCCATCTTCCCACCATCC-3' and 5'TCTCGCGGCCGCTGTCTCTAACACTCATTCCTG-3'. The pTS-mIgG and pTS-mIgK plasmids were constructed by replacing the human IgG constant gene of pTS-hIgG with the PmlI/NotI-digested mouse IgG or IgK constant region, respectively. A linear pNS-mIgG, a non-selective vector, was amplified from pTS-mIgG by PCR with the primers MCSAP3-AS and mIgG2nd-S (5'- TGCCTGGT CAAGGGCTATTTCCCTGAG-3').

\section{Red/ET-mediated recombination}

Red/ET-mediated recombination was carried out as described previously [15]. Vectors were linearized outside of the homology region by digestion with EcoRV. The linear vector $(0.1 \mu \mathrm{g})$ and the PCR-amplified DNA were mixed in a 1:2 molar ratio and transformed into competent cells. After recombination, bacteria were plated on agar plates containing $1 \%$ sucrose and $50 \mu \mathrm{g} / \mathrm{ml}$ kanamycin or directly inoculated into LB medium containing $1 \%$ sucrose and $50 \mu \mathrm{g} / \mathrm{ml}$ kanamycin.

\section{Colony PCR}

Bacterial colonies were suspended in $100 \mu \mathrm{l}$ of PBS-0.1\% Triton X-100 solution and heated at $95^{\circ} \mathrm{C}$ for 5 minutes to extract the plasmid DNA. For PCR, $10 \mathrm{pmol}$ of primers (MCSAP3-S and 618/642-AS) was added to $1 \mu \mathrm{l}$ of the above cell-body heat-extracted solution, and PCR was performed in $50 \mu \mathrm{l}$ of a reaction system using the ExTaq DNA polymerase (30 cycles of reaction at $94^{\circ} \mathrm{C}$ for 30 seconds and $68^{\circ} \mathrm{C}$ for 40 seconds).

\section{Sequence analysis}

Nucleotide sequences were determined with an Applied Biosystems 373 DNA sequencer using a BigDye Terminator v.3.1 Cycle Sequence Kit (Applied Biosystems). The $\mathrm{V}$-(D)-J sequences were aligned with IMGT/V-QUEST (http://imgt.cines.fr/IMGT_vquest/share/textes/)[17].

\section{Expression of recombinant mouse antibodies}

5' RACE-amplified mouse VH and VL genes were cloned in-frame into pTS-mIgG and pTS-mIgK using 
TS-HR methodology. Plasmids were purified with PureYield Plasmid Miniprep kits (Promega). Cognate pairs of $\mathrm{VH}$ and VL genes were co-transfected into 293FT cells grown in 24-well culture dishes with FuGENE HD Transfection Reagent (Roche). Two days after transfection, cell culture supernatants were analyzed for the secretion of recombinant antibodies. The antibody concentration and antibody reactivity were determined by a sandwich enzyme-linked immunosorbent assay (ELISA). Briefly, high-binding-capacity ELISA plates (Corning) that were pre-coated with either goat anti-mouse IgG (Sigma) or egg albumin were incubated for $1 \mathrm{~h}$ with 200 $\mu \mathrm{l}$ of DMEM containing 10\% FBS for blocking. After washing the plates $3 \mathrm{x}$ with PBS, the cell-culture supernatant was transferred into the ELISA plates and incubated for $2 \mathrm{~h}$ at room temperature. After washing the plates with PBS, $100 \mu \mathrm{l}$ of alkaline phosphatase (AP)conjugated goat anti-mouse IgG (Sigma) at a concentration of $0.8 \mu \mathrm{g} / \mathrm{ml}$ in PBS was added to the wells and incubated for $2 \mathrm{~h}$. After washing with PBS, the assays were developed using BM Chemiluminescence ELISA Substrate (AP) (Roche) and quantified with a Tecan GENios microplate reader (TECAN, Crailsheim, Germany). The magnitude of the light emission was expressed as relative light units (RLU).

\footnotetext{
Abbreviations

TS-HR: target-selective homologous recombination; $\mathrm{V}$ : immunoglobulin variable; VL: immunoglobulin light chain variable; VH: immunoglobulin heavy chain variable; 5' RACE: rapid amplification of 5' CDNA ends; ELISA: enzymelinked immunosorbent assay; AP: alkaline phosphatase; IgG: immunoglobulin gamma; RT-PCR: reverse-transcription polymerase chain reaction; RLU: relative light unit; PCR: polymerase chain reaction
}

\section{Acknowledgements}

This research was supported by grants from Hokuriku Innovation Cluster for Health Science.

\section{Author details \\ ${ }^{1}$ Laboratory of Molecular and Cellular Biology, Faculty of Science and Engineering, Graduate School, University of Toyama, 3190 Gofuku, Toyama- shi, Toyama, 930-8555, Japan. ${ }^{2}$ Graduate School of Innovative Life Science, University of Toyama, Toyama-shi, Toyama, 930-8555, Japan. ${ }^{3}$ Laboratory of Molecular and Cellular Biology, Faculty of Science and Engineering, Graduate School, University of Toyama, 3190 Gofuku, Toyama-shi, Toyama, 930-8555, Japan.}

\section{Authors' contributions}

NK designed and performed the experiments and drafted the manuscript. MY performed the experiments and analyzed the data described in this study. Ml organized the experiments. All authors read and approved the final manuscript.

Received: 4 February 2011 Accepted: 13 April 2011

Published: 13 April 2011

\section{References}

1. Babcook JS, Leslie KB, Olsen OA, Salmon RA, Schrader JW: A novel strategy for generating monoclonal antibodies from single, isolated lymphocytes producing antibodies of defined specificities. Proc Natl Acad Sci USA 1996, 93(15):7843-7848.
2. Doenecke A, Winnacker EL, Hallek M: Rapid amplification of CDNA ends (RACE) improves the PCR-based isolation of immunoglobulin variable region genes from murine and human lymphoma cells and cell lines. Leukemia 1997, 11(10):1787-1792.

3. Wrammert J, Smith K, Miller J, Langley WA, Kokko K, Larsen C, Zheng NY, Mays I, Garman L, Helms C, et al: Rapid cloning of high-affinity human monoclonal antibodies against influenza virus. Nature 2008, 453(7195):667-671

4. Coronella JA, Telleman P, Truong TD, Ylera F, Junghans RP: Amplification of IgG VH and VL (Fab) from single human plasma cells and B cells. Nucleic Acids Res 2000, 28(20):E85.

5. Tiller $\mathrm{T}$, Busse $\mathrm{CE}$, Wardemann $\mathrm{H}$ : Cloning and expression of murine Ig genes from single B cells. J Immunol Methods 2009, 350(1-2):183-193.

6. Tiller T, Meffre E, Yurasov S, Tsuiji M, Nussenzweig MC, Wardemann H: Efficient generation of monoclonal antibodies from single human $\mathrm{B}$ cells by single cell RT-PCR and expression vector cloning. J Immunol Methods 2008, 329(1-2):112-124.

7. Landy A: Dynamic, structural, and regulatory aspects of lambda site-specific recombination. Annu Rev Biochem 1989, 58:913-949.

8. Kitts PA, Nash HA: Bacteriophage lambda site-specific recombination proceeds with a defined order of strand exchanges. J Mol Biol 1988, 204(1):95-107.

9. Liu Q, Li MZ, Leibham D, Cortez D, Elledge SJ: The univector plasmidfusion system, a method for rapid construction of recombinant DNA without restriction enzymes. Curr Bio/ 1998, 8(24):1300-1309.

10. Hamilton MD, Nuara AA, Gammon DB, Buller RM, Evans DH: Duplex strand joining reactions catalyzed by vaccinia virus DNA polymerase. Nucleic Acids Res 2007, 35(1):143-151.

11. Jones ML, Seldon T, Smede M, Linville A, Chin DY, Barnard R, Mahler SM, Munster D, Hart D, Gray PP, et al: A method for rapid, ligationindependent reformatting of recombinant monoclonal antibodies. $\mathrm{J}$ Immunol Methods 2010, 354(1-2):85-90.

12. Zhang $Y$, Muyrers JP, Testa G, Stewart AF: DNA cloning by homologous recombination in Escherichia coli. Nat Biotechnol 2000, 18(12):1314-1317.

13. Zhang $Y$, Buchholz F, Muyrers JP, Stewart AF: A new logic for DNA engineering using recombination in Escherichia coli. Nat Genet 1998, 20(2):123-128.

14. Muyrers JP, Zhang Y, Buchholz F, Stewart AF: RecE/RecT and Redalpha/ Redbeta initiate double-stranded break repair by specifically interacting with their respective partners. Genes Dev 2000, 14(15):1971-1982.

15. Fujimoto R, Osakabe T, Saito M, Kurosawa N, Isobe M: Minimum length of homology arms required for effective Red/ET recombination. Biosci Biotechnol Biochem 2009, 73(12):2783-2786.

16. Frohman MA, Dush MK, Martin GR: Rapid production of full-length cDNAs from rare transcripts: amplification usig a single gene-specific oligonucleotide primer. Proc Natl Acad Sci USA 1988, 85(23):8998-9002.

17. Brochet $X$, Lefranc MP, Giudicelli V: IMGT/V-QUEST: the highly customized and integrated system for IG and TR standardized V-J and V-D-J sequence analysis. Nucleic Acids Res 2008, 36(Web Server):W503-508.

doi:10.1186/1472-6750-11-39

Cite this article as: Kurosawa et al:: Target-selective homologous recombination cloning for high-throughput generation of monoclonal antibodies from single plasma cells. BMC Biotechnology 2011 11:39.

\section{Submit your next manuscript to BioMed Central and take full advantage of:}

- Convenient online submission

- Thorough peer review

- No space constraints or color figure charges

- Immediate publication on acceptance

- Inclusion in PubMed, CAS, Scopus and Google Scholar

- Research which is freely available for redistribution 